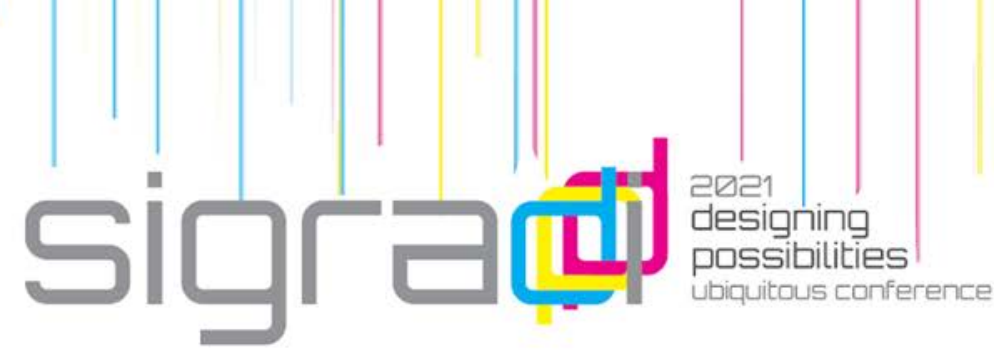

\title{
Wedged Kerfing. Design and Fabrication Experiments in Programmed Wood Bending
}

\author{
Roberto Naboni ${ }^{1}$, Salvatore Dario Marino ${ }^{2}$ \\ ${ }^{1}$ CREATE Group, University of Southern Denmark, Denmark \\ ron@iti.sdu.dk \\ 2 Università degli Studi di Pavia, Italy \\ CREATE Group, University of Southern Denmark, Denmark \\ salvatoredario.marino@unipv.it
}

\begin{abstract}
Wood as a construction material gained interest in the last decade because of its low carbon footprint and the continuous research of new design possibilities opened using computational and robotic means. The shaping of timber into nonstandard shapes is challenging and industrially demanding. This paper showcases a method to computationally control the formation of curved wood elements suitable for construction purposes. The aim is to achieve wood bending and twisting through a technique that combines advanced kerfing with a controlled insertion of wedges. The research has been conducted through material testing, computational developments, and robotic prototyping to evaluate design control, fabrication accuracy, and structural potential for architectural applications.
\end{abstract}

Keywords: Robotic fabrication, Wood construction, Wedged Kerfing, Wood Bending

\section{Introduction}

Wood is the only effective construction material that is truly renewable and that, by its very essence, has structural properties for architectural purposes. The development of new technical solutions in the wood industry - including material engineering, novel manufacturing methods, and lamination techniques - is promoting a focus on wood as a carbon-efficient alternative for contemporary and future construction (Svilans et al., 2019). The diffusion of computational methodologies is expanding the effective design space and the level of complexity that can be achieved with timber. In recent years, major efforts have been dedicated to implementing robotic assembly procedures (Naboni et al. 2021, Kunic et al. 2021a, Kunic et al. 2021b). However, consolidated 
manufacturing processes in the industry have been poorly developed to meet the increasing needs for customization. For instance, the realization of curved wood elements can still be challenging with existing techniques based on mass production, and require inflexible, time-consuming, and expensive developments, often involving highly specialized consulting firms in both the design and manufacturing phases (Hua et. al, 2021). With the more general aim of exploring digitally led alternatives to contemporary production methods, this research work proposes an approach to the production of curved structural timber elements through programmed machining patterns. Implementing the craft technique of kerfing, we developed a robust digital workflow to design, and robotically fabrication curved wood elements of variable curvature, and evaluated fabrication and structural properties.

\subsection{Background}

Kerfing is a technique of patterned cutting that allows rigid material to be bent (Satterfield et al., 2020). The removal of material, performed through a cutting process using tools such as a circular saw or a milling bit, is used to locally reduce locally the material section and obtain living hinges. The bending of a wood element is therefore achieved by the effect of consecutive living hinges which rotate around the kerf axes.

Historically, kerfing has been used to bend wood sheets and linear elements in furniture making and the production of musical instruments, i.e., guitars. Explorations in product design used cut-through patterns that were applied through lasers to deform planar sheets (Capone, 2018). Recent research at the architectural scale was using kerfing to obtain elastic bending in the installation of Gramazio \& Kohler for Dukta exhibition and the Intermediate Fragment (Aagaard, 2017), and to counterbalance tensional elements in the Kerf-based Complex Assemblies (Menges et al., 2011). In these works, kerfed wood battens achieve flexibility through an intentional material weakening, and this would prevent their use for structural applications unless coupled with other structural elements. (Satterfield et al., 2019) have experimented with zipping, a variation of the kerfing technique introduced by (Schindler, 2008), where two elements are reciprocally bent into position and glued to achieve a plastic deformation with some structural capacity. However, this technique requires a relatively complex fabrication process that ends up with nearly half of the initial material lost in machining.

Exploring structural alternatives, while minimizing material waste and enhancing production efficiency, we introduce the Wedged Kerfing, a technique that uses the progressive insertion of wood wedges obtained from production leftovers to achieve rigid bent battens (Figure 1). This paper describes material experiments, computational developments, robotic prototyping and evaluates the suitability of this approach for architectural applications. 


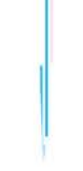

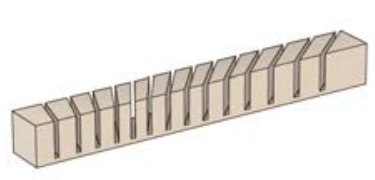

Kerfed batten

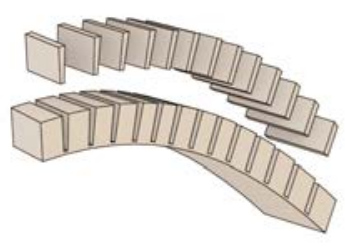

Wedges insertion

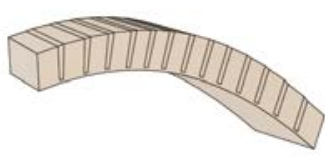

Wedged kerf-bent batten

Figure 1. Wedges insertion in a kerfed batten. Source: author.

\section{Methodology}

The research methodology for this work is mainly based on computational modelling and prototyping experiments with robotic kerfing to bend wood battens. The controlled bending and twisting of timber battens are obtained from a computational workflow and the manufacturing of elements that have a potential for structural applications.

\subsection{Preliminary Material Experiments}

Two preliminary experiments have been performed to understand material behaviour and define the main fabrication constraints for this technique. Firstly, the kerf-bending behaviour of a timber batten induced by the insertion of wedges was evaluated by comparing two distinct kerfing methods. The elastic bending of a batten can be in fact obtained by both kerfing its tension or the compression side. We name these two methods as expansion kerfing and compression kerfing, respectively. The maximum wedge angle formed by two kerf sides after bending was measured to determine the achievable bending radius of a batten. The experiment was conducted on pinewood battens with a sectional height of $38 \mathrm{~mm}$ and moisture content of $25 \%$, by applying evenly distributed cuts with a $30 \mathrm{~mm}$ interval perpendicular to the direction of the fibers (Figure 2).

Wedge angles of $2^{\circ}, 4^{\circ}, 6^{\circ}, 8^{\circ}, 10^{\circ}$ were tested on a new series of battens. We observed signs of cracking starting from a $6^{\circ}$ angle in the expansion kerfing, while the compression kerfing began to crack at $8^{\circ}$. With both approaches it was possible to achieve a wedge angle of $10^{\circ}$ by soaking the wood in water for a few minutes before the bending test. Expansion kerfs, however, do not require the use of clamps to hold the piece in position, but are more challenging to glue evenly when the angle increases (Figure 3 ). 

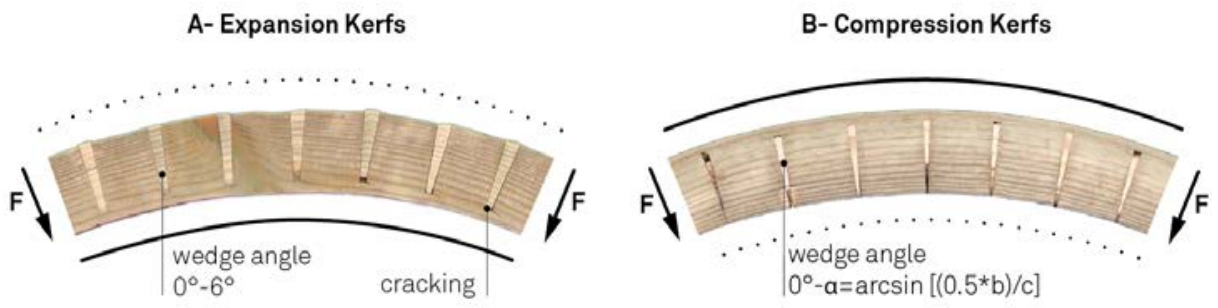

Figure 2. Comparison of expansion and compression kerfs. Source: author.
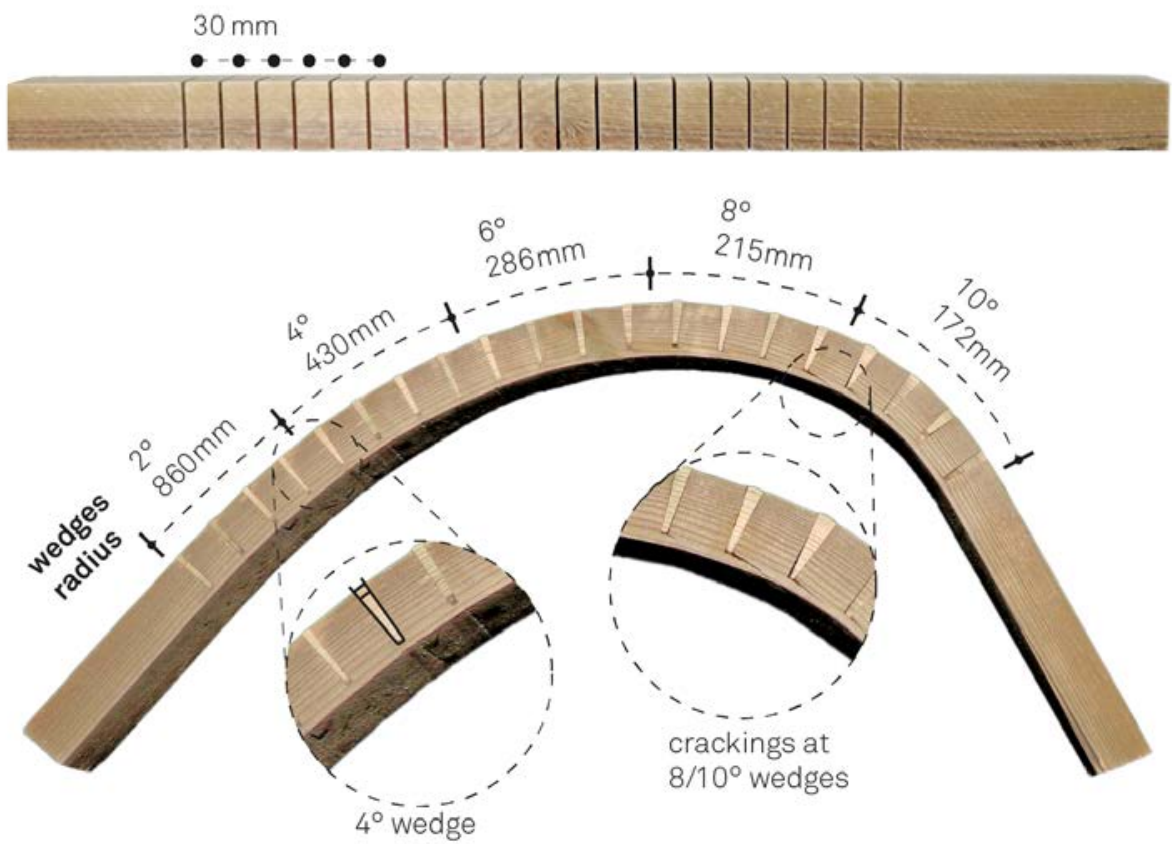

Figure 3. Experiment on wedge angles and achievable bending radios. Source: author.

The second experiment was conducted to explore the twisting behaviour of kerfed battens due to the variation of the kerf angle, defined as the angle formed by a saw cut and the perpendicular to the batten's primary axis along the orientation of the fibres, measured on the horizontal plane. The kerf cuts were marked on the batten's lateral face with a regular distance of $30 \mathrm{~mm}$, which means the larger the angle, the closer the kerfs would get. Four cuts were applied for each of the angle series $0^{\circ}, 15^{\circ}, 30^{\circ}, 45^{\circ}, 60^{\circ}, 75^{\circ}$. With a constant wedge angle of $4^{\circ}$, there was no observed material failure. However, while 

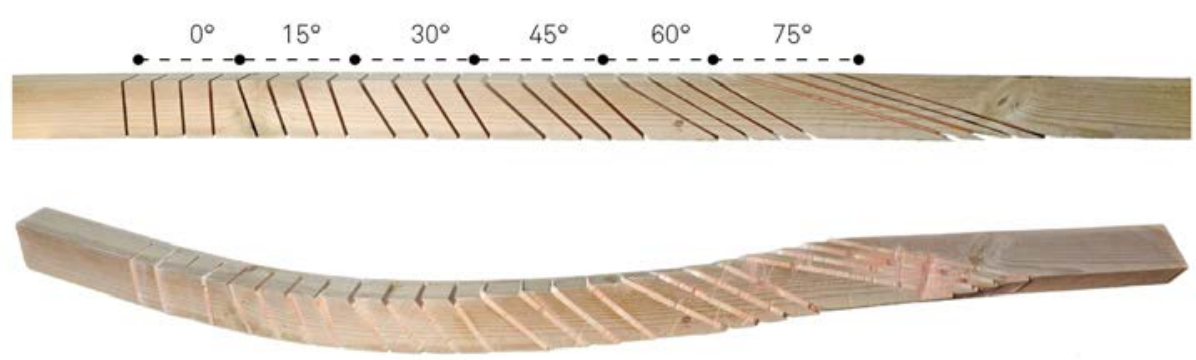

Figure 4. Experiment on variable kerf angles and batten twisting. Source: author.

larger angles pose some geometrical challenges in the insertion of wedges, they are more easily bending as they progressively align with the wood fibers (Figure 4).

\subsection{Computational Workflow for Wedged-Kerfing}

Based on the preliminary material experiments, a computational workflow for wedged kerfing design and fabrication has been developed, which allows the rationalization of free-form input curves into precisely bent battens (Figure 5).

(i) Initially it is defined an Input Geometry - a target curve or a polyline that is used as design target. This is quickly reconstructed as a 3D elastica curve that behaves like an elastic rod and minimizes sudden discontinuities in the curvature.

(ii) Physics-based Developability - The elastica curve is used to model an approximate surface geometry, then used to generate a developable surface. We implemented a physics-based form-finding process in Kangaroo for Grasshopper to obtain such a geometry with a mean gaussian curvature $k=0$. Forces are applied to the vertices of the quad mesh to obtain a set of consecutive planar mesh faces, which can be successfully unrolled without distortion on the XY plane.

(iii) Rulings Generation and Angles Equalization - The vertices of the bent mesh with planar quads are extracted and interpolated into separated edge curves, which define two opposite edges of the batten. The rulings of the developable surface are computed by connecting pairs of points with a corresponding tangent along the two curves (Satterfield et al., 2019). To ease the assembly process, the wedge angles are set to $5^{\circ}$, while their distribution across the batten is parametrized to achieve the desired bending ratio. This process is completed with a recursive loop implemented in Anemone for Grasshopper, which 


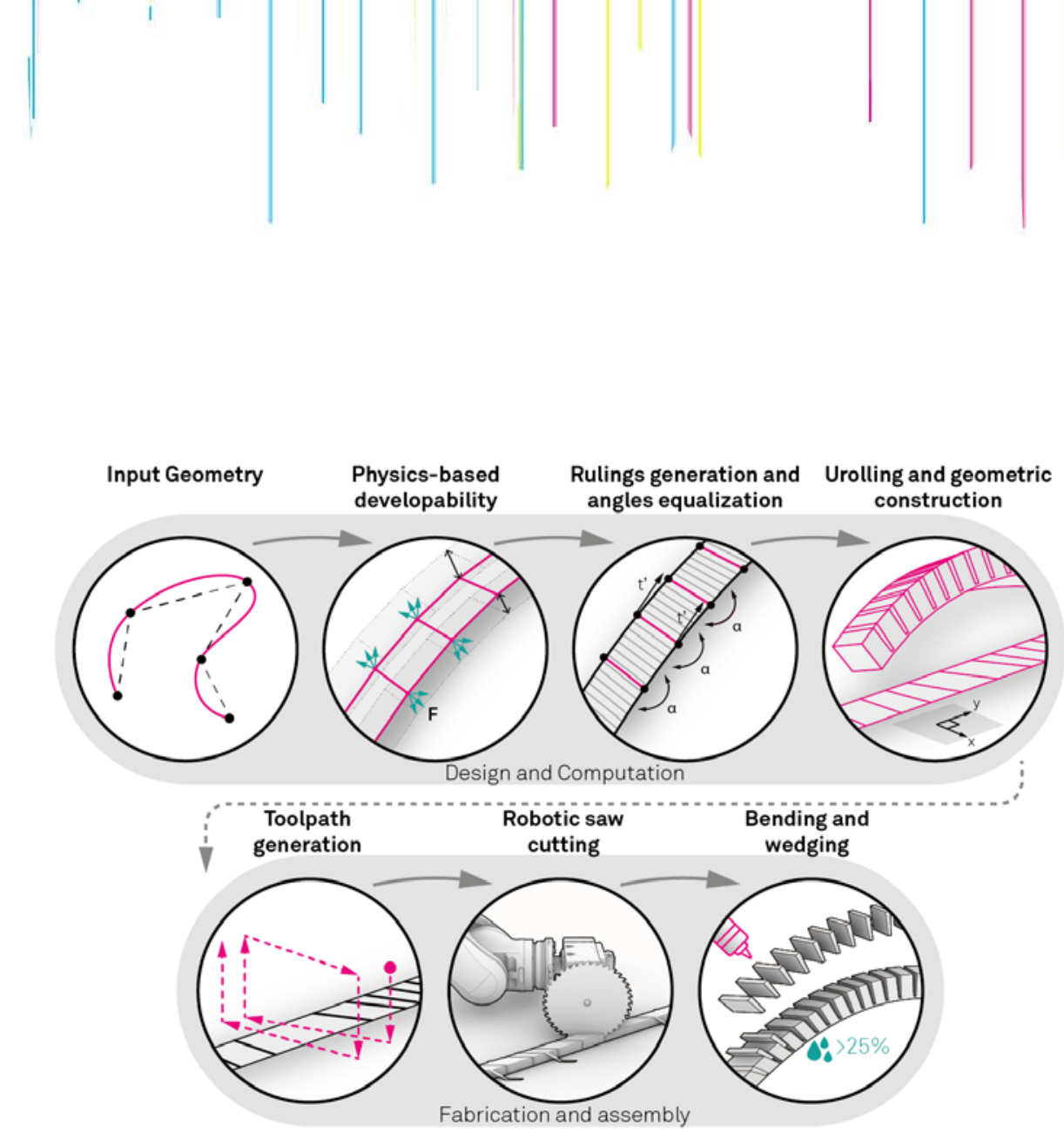

Figure 5. Computational workflow. Source: author.

outputs a set of rulings that correspond that are afterwards used as rotation axis, around which is used a constant angle $(\alpha)$ to achieve a controlled bending deformation.

(iv) Unrolling and Geometric Construction - the obtained surface with equalized angles is subsequently unrolled onto the $X Y$ plane to generate a $3 \mathrm{D}$ component model to be manufactured through simple geometric constructions. This model can be used for both visualization and FEA simulation.

\subsection{Robotic Fabrication and Assembly}

Linked to the design phase, the fabrication and assembly process is performed consisting of:

(v) Toolpath Generation - starting from the previously unrolled geometry, rulings trajectories are used to generate a continuous toolpath for robotic sawing, where cutting speed and direction, approaching ramps, and in-between motions are generated. In this phase are also established the key coordinates for the installation of custom-designed clamps.

(vi) Robotic Saw-Cutting - A $3.8 \mathrm{~mm}$ thick saw blade with a $350 \mathrm{~mm}$ wide diameter was mounted on a Kuka KR 240 r3330 equipped with a milling spindle. The working area for the robot was set up on a $4.5 \mathrm{~m}$ long welding table. The battens were fixed to a wood board through a 


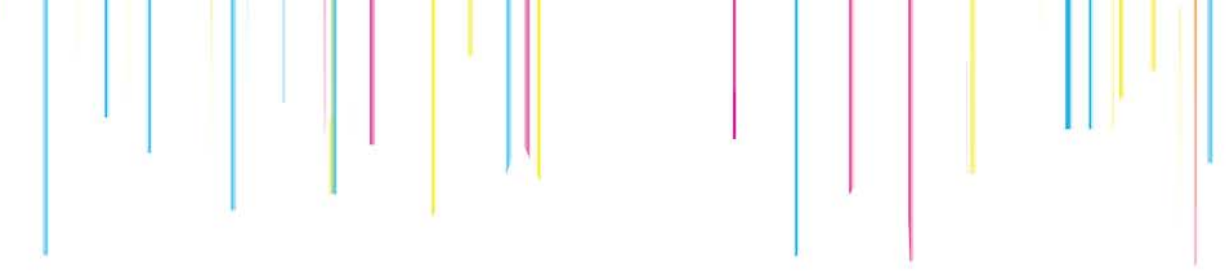

system of custom-made steel brackets, which are screwed to the tabletop following safe marks positioned according to coordinates materialized by the robot (Figure 6).

(vii) Bending and Wedging - in parallel, trapezoidal wedges of an equal size were mass-produced manually with a table bandsaw from production leftovers. Once the robotic sawing of the batten is completed, the wood is soaked into water for a few minutes to increase the relative moisture content. The battens are then bent into position with the insertion of the wood wedges, which are applied along with glue.

\section{$3 \quad$ Results}

The results of this process are observed in terms of bending capacity, fabrication accuracy, and structural integrity. Material experiments indicate that the bending capacity of wedged kerfing is determined by the maximum wedge angle achievable with a specific material. In the pinewood utilized in this work, angles between $0^{\circ}-5^{\circ}$ were successfully used with both expansion and compression kerfing, without observing any material failure. To enhance the fabrication efficiency and precision, the wedge angle was standardized to $5^{\circ}$ degrees. Through the described computational design process can be a determined the bending and twisting of wood battens by programmed variations of the kerfing angles and the distribution of the kerf counts (Figure 7).

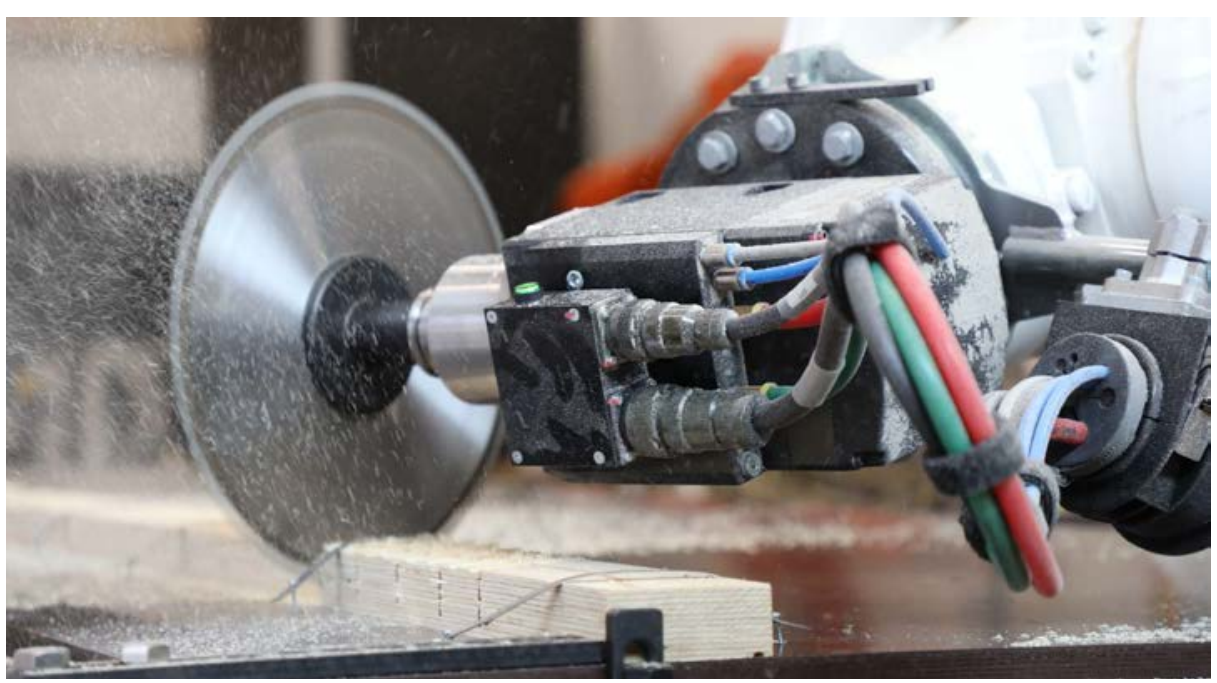

Figure 6. Robotic fabrication set-up with a $3.8 \mathrm{~mm}$ thick and $350 \mathrm{~mm}$ diameter saw blade mounted on a Kuka KR 240 r3330. Source: author. 

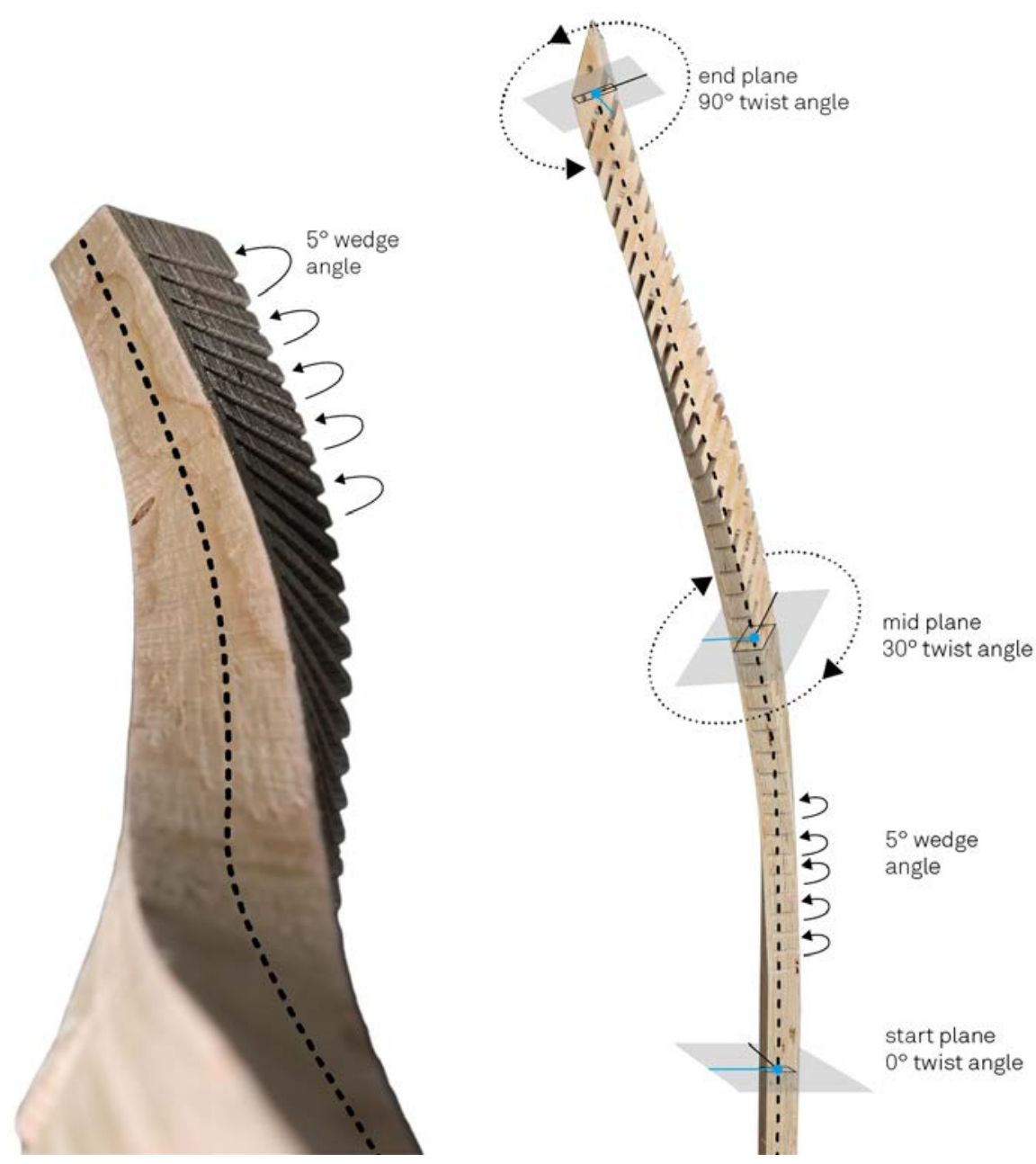

Figure 7. Realized prototype and visualization of the twisting angle and frames at the batten's start, middle and end. Source: author.

A large variety of bending and twisting radios were achieved in both the computational model and in physical prototypes for both the method of expansion and compression wedge kerfing (Figure 8).

Fabrication Accuracy - The realized prototypes were 3D scanned through photogrammetry to evaluate the deviation from the achieved physical artefacts and the $3 \mathrm{~d}$ model. The point cloud analysis showed displacements in the range $40-140 \mathrm{~mm}$ for battens of $1500 \mathrm{~mm}$ in length (Figure 9). 


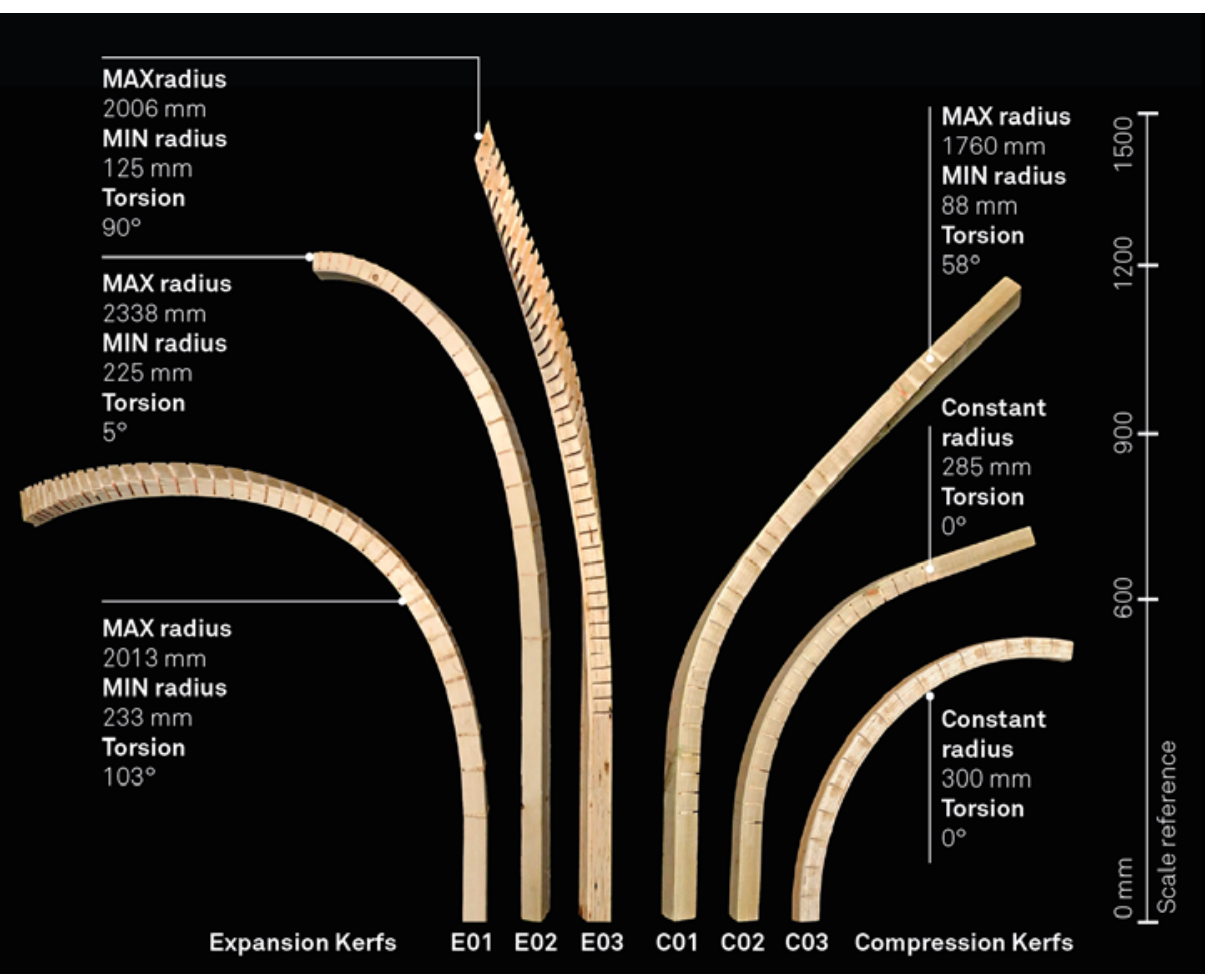

Figure 8. Realized prototypes, left with expansion kerf, right with compression kerfs. Source: author.

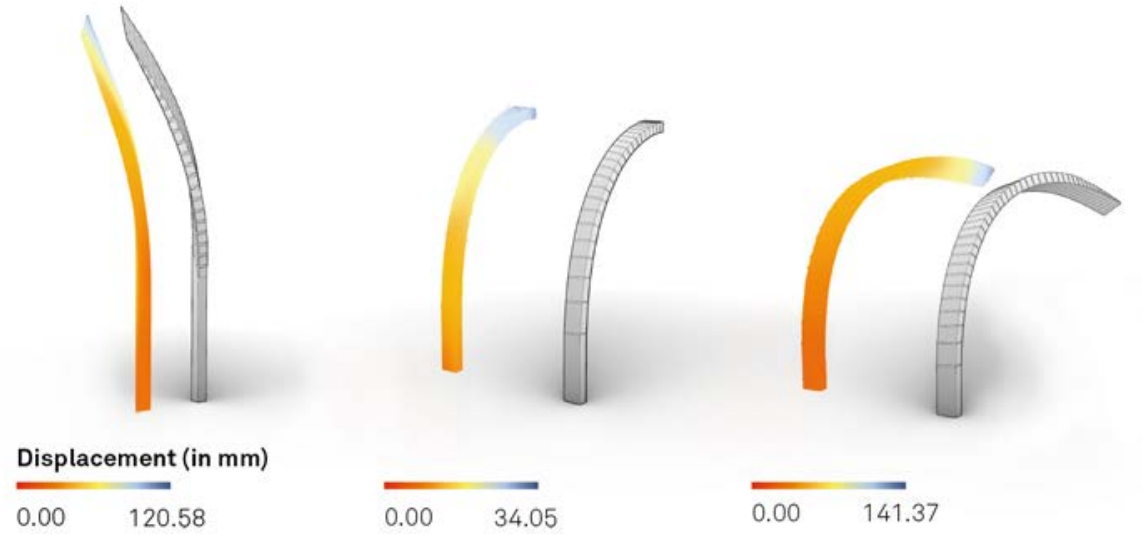

Figure 9. Model of bent and twisted battens and comparison with the 3D photogrammetric scanning of the fabricated one. Source: author. 


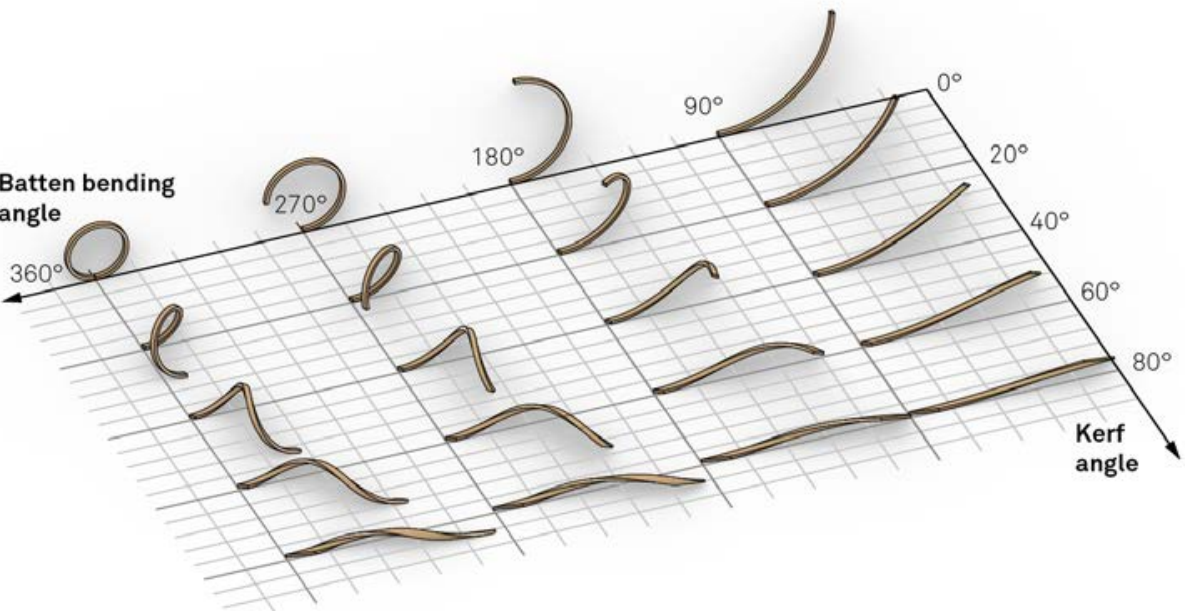

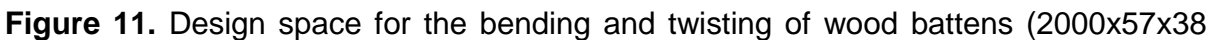
$\mathrm{mm}$ ). The combined variation of the batten's global bending angle and kerf angle shows a wide set of possibilities. Source: author.

fabrication experiments. This early study focused on exploring the potential of this technique for the mouldless production of curvilinear structures. Based on the presented results, this approach shows a potential for the realization of a large variety of bending and twisting shapes, which would be very complex to manufacture with traditional techniques (Figure 11).

Further material studies shall be conducted in future to improve the system geometrical accuracy, investigate the structural response with a series of mechanical laboratory tests, and study the up scaling of this proof of concept on larger timber elements for the use in real-life structures.

Acknowledgements. The authors wish to thank Hamed Hajikarimian for his support in the FEM presented in the results section of this paper. 


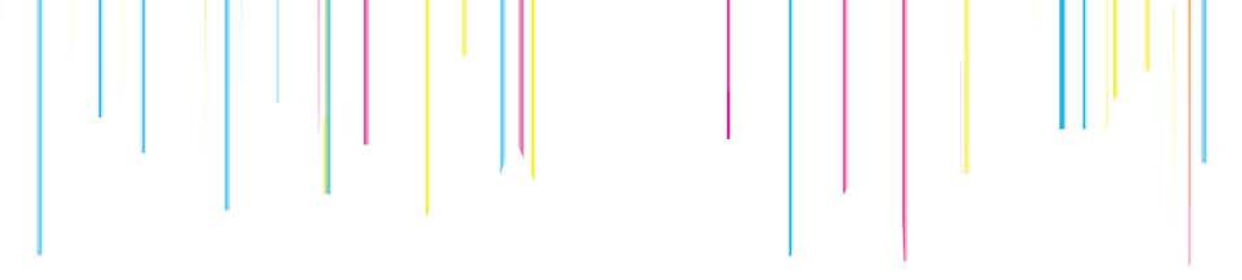

\section{References}

Chai, H., So, C., \& Yuan, P. F. (2021). Manufacturing double-curved glulam with robotic band saw cutting technique. Automation in Construction, 124(1239). https://doi.org/10.1016/j.autcon.2021.103571

Capone, M., \& Lanzara, E. (2018). Kerf bending: ruled double-curved surfaces manufacturing. November, 653-660. https://doi.org/10.5151/sigradi2018-1389

Kruse Aagaard, A. (2017). Bespoke Fragments: Materials and digital fabrication in architectural design. Arkitektskolens Forlag.

Kunic, A., Naboni, R., Kramberger, A., \& Schlette, C. (2021). Design and assembly automation of the Robotic Reversible Timber Beam. Automation in Construction, 123(December 2020), 103531. https://doi.org/10.1016/j.autcon.2020.103531.

Kunic, A., Kramberger, A. and Naboni, R. (2021) Cyber-Physical Robotic Process for Re-Configurable Wood Architecture. Closing the circular loop in wood architecture. Proceedings of the 39th eCAADe Conference. Towards a new, configurable architecture. Novi Sad, September 2021.

Menges, A. (2011). Integrative Design Computation. Acadia, 72-81.

Mitov, D., Tepavčević, B., Stojaković, V., \& Bajšanski, I. (2019). Kerf Bending Strategy for Thick Planar Sheet Materials. Nexus Network Journal, 21(1), 149-160. https://doi.org/10.1007/s00004-018-0415-7

Naboni, R., Kunic, A., Kramberger, A., \& Schlette, C. (2021). Design, simulation and robotic assembly of reversible timber structures. Construction Robotics, 5(1), 1322. https://doi.org/10.1007/s41693-020-00052-7

Schindler, C. (2008). A Computer-Aided Fabrication Method for Bending Panels without Molds. Session 19: Generative Design - ECAADe 26, Figure 1, 795^802. http://papers.cumincad.org/data/works/att/ecaade2008_018.content.pdf

Satterfield, B., Preiss, A., Mavis, D., \& Entwistle, G. (2019). Twisted Logic | Thinking Outside and Inside the Box. Black Box 107th ACSA Annual Meeting, 333-340.

Satterfield, B., Preiss, A., Mavis, D., \& Entwistle, G. (2019). Zippered Wood : Small Material Moves can Bend Large Systems. ACADIA 2019 - Ubiquity and Autonomy, 116-121.

Satterfield, B., Preiss, A., Mavis, D., Entwistle, G., Swackhamer, M., \& Hayes, M. (2020). Bending the Line: Zippered Wood Creating Non Orthogonal Architectural Assemblies Using the Most Common Linear Building Element Component (The 2x4). Fabricate 2020, Making Resilient, 58-65

Svilans, T., Tamke, M., Thomsen, M. R., Runberger, J., Strehlke, K., \& Antemann, M. (2019). New workflows for digital timber. In Lecture Notes in Civil Engineering (Vol. 24). Springer International Publishing. https://doi.org/10.1007/978-3-030-036768_3 